\title{
Receptor-based predictors of response in breast cancer
}

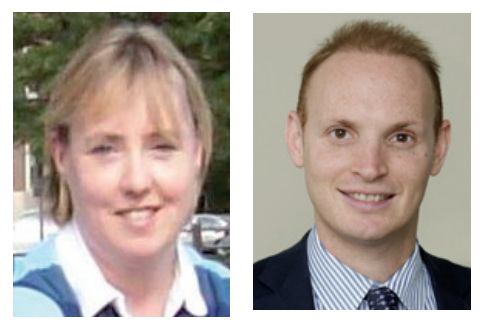

"Further biomarkers are needed if we are to truly realize the potential for improving our prognostic and predictive tools in the treatment of breast cancer."

\section{Ursula B McGovern† \& Justin Stebbing}

†Author for correspondence: Imperial College Healthcare NHS Trust, Charing Cross Hospital.

Department of Medical Oncology, 1st Floor, East Wing, Fulham Palace Road, London, UK

n Tel. : +44 2087468295 n Fax: +44 2088461433 n u.mcgovern@imperial.ac.uk

Breast cancer remains the most common malignancy in women and mortality rates are falling, but are we really making inroads into the treatment of this complex and heterogeneous disease? Decision making regarding chemotherapy relies on a predictable but often flawed set of criteria, which oncologists have used for the last 20 years - axillary lymph node status, tumor size, lymphovascular invasion, grade and hormone receptor status. There is little doubt that with the advent of new biological and targeted treatments and the development of new molecular techniques, we are still failing to clearly identify those patients who will benefit from chemotherapy, whilst on the other hand heavily overtreating individuals who may derive little benefit from systemic therapy. Improved tools are required to predict response to treatment in breast cancer.

It is over 30 years since growth receptors were first identified and their expression in breast cancer still correctly plays a pivotal role in treatment decisions. Evaluating the expression of hormone receptors and HER2 expression may now be routine, but what information does this provide us with in regards to the likelihood of response to treatment, and what new biomarkers can be used to further improve our treatment selections?

The estrogen receptor (ER) allows us to predict, at least to some extent, the response of breast cancer to endocrine treatment. Patients with tumors that are also strongly positive for the progesterone receptor $(\mathrm{PgR})$ have the highest likelihood of response [1]. We now know that ER and/or PgR expression is an independent prognostic factor in breast cancer [2]. However, in terms of benefit from chemotherapy, it appears that ER-negative tumors tend to respond better to chemotherapy, rather than ER-positive tumors. Studies have also shown that the likelihood of achieving a pathological complete response (pCR) with primary chemotherapy, a good surrogate for long-term survival in the neoadjuvant setting, is also significantly higher in ER-negative tumors [3]. Thus, patients who have ER-negative cancers likely derive the most benefit from adjuvant chemotherapy.

Overexpression of HER2 is observed in 20-30\% of breast cancers, and this has been associated with more aggressive tumors. At least two different genetic mechanisms can lead to increased HER2 copy number - gene amplification and aneuploidy, where a change in the number of extra copies of chromosome 17 (polysomy 17) is seen [4]. Polysomy 17-positive tumors, which lack HER2 gene amplification, are pathologically indistinguishable from HER2-negative tumors, and although they have extra copies of HER2, they are very different from tumors in which the HER2 gene is amplified, and may not respond to HER2-targeted therapies.

We can use HER2 expression as a predictor of response to chemotherapy. Some studies have shown that patients who have HER2-positive tumors will benefit from an anthracyclinecontaining chemotherapy regimen, although the exact mechanism for this remains unclear. There is close proximity of the HER2 gene to the TOP $2 A$ gene located on chromosome $17 \mathrm{q}$, and topoisomerase II is known to be a target of the anthracyclines, amongst other proteins [5]. A number of studies have suggested that deletion or overamplification of TOP $2 \mathrm{~A}$, perhaps closely associated with coamplification of HER2, leads to a worse prognosis but greater response to anthracycline-containing regimens. Therefore, these are potentially the patients that we should be identifying as requiring aggressive treatment with an anthracycline-containing regimen.

Conversely, HER2 positivity may also be associated with a benefit from treatment with paclitaxel. A study involving 1500 women with

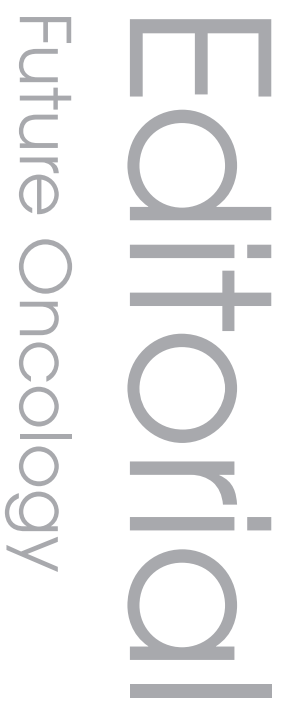


node-positive breast cancer demonstrated a significant interaction between HER2 status and the addition of adjuvant paclitaxel in patients who had received four cycles of doxorubicin plus cyclophosphamide. This interaction was associated with a hazard ratio for recurrence of 0.59 $(\mathrm{p}=0.01)^{[6]}$. This was independent of ER status: no such benefit was seen with HER2-negative, ER-positive breast cancers.

HER2 overamplification also raises the issue about the use of endocrine treatment. Whilst the debate continues about sequential or extended endocrine treatment in ER-positive patients, is tamoxifen really redundant in HER2-positive patients? Data from the P24 trial comparing neoadjuvant letrozole with tamoxifen demonstrated that in the neoadjuvant setting the response of HER2-positive tumors to tamoxifen was significantly lower than that of HER2-negative tumors - 88 versus $21 \%(\mathrm{p}=0.0004)[7]$. Similar results were seen in the neoadjuvant Immediate Preoperative Anastrozole, Tamoxifen, or Combined With Tamoxifen (IMPACT) trial, where a response rate of $58 \%$ was observed in the anastrozole arm compared with $22 \%$ for tamoxifen in HER2-positive tumors. However these data were not statistically significant, as the analysis was underpowered due to small numbers of patients [8]. There is cross-talk between ER and growth factor receptors, with cytoplasmic ER functioning as a growth receptor ligand, activating HER2 tyrosine kinase activity [9]. As these two receptor systems have the ability to activate each other, combined targeting of both receptors appears to be an attractive therapeutic option.

What of some of the newer receptors identified - will they provide useful markers in the future for response to treatment, particularly targeted treatments? Studies have shown that the EGF receptor (EGFR/HER1) is overexpressed in $16-48 \%$ of breast cancers, depending on how it is measured [10]. Targeting EGFR remains an attractive therapeutic option. This includes the use of monoclonal antibodies such as cetuximab and small-molecule tyrosine kinase inhibitors, such as gefitinib and erlotinib. However, studies have shown that the presence of EGFR per se is a poor marker of response, and trials investigating the use of both gefitinib and erlotinib in breast cancer have been disappointing. What has emerged, particularly in the treatment of non-small-cell lung cancer, is the observation that specific mutations in the EGFR tyrosine kinase domain may in fact predict response to
EGFR inhibitors. The discovery of missense and deletion mutations may lead to a higher affinity of EGFR for gefitinib, and thus lead to a better response to the drug [11]. However, mutations have not been found here in breast cancer, and as expression of EGFR does not equate to a response to EGFR inhibition, the therapeutic options may be limited. Results with lapatinib in breast cancer have been more encouraging [12]. Lapatinib, a dual tyrosine kinase inhibitor, targets both EGFR and HER2, and a number of trials are underway investigating it in EGFRpositive HER2-negative disease to further understand the role of the EGFR.

\footnotetext{
"Whilst the debate continues about sequential or extended endocrine treatment in ER-positive patients, is tamoxifen really redundant in HER2-positive patients?"
}

Invasive breast cancers that overexpress VEGF have a worse clinical outcome and are often resistant to both hormone therapy and chemotherapy [13]. Bevacizumab has been shown to have activity in advanced breast cancer - its addition to paclitaxel improved response rates from $14 \%$ for paclitaxel alone to $28 \%$ with the combination [14]. In advanced breast cancer it is likely that a number of proangiogenic factors are in operation, and targeting a single pathway is a fruitless operation. VEGF overexpression appears to be an early step in breast cancer progression and is seen frequently in ductal carcinoma in situ, long before tumor invasion [15]. By the time patients have developed metastatic disease, the benefits of bevacizumab may be lost, and we are now undertaking trials in early-stage disease to truly assess the benefit of bevacizumab and its role in the adjuvant setting. Sunitinib and sorafenib, both tyrosine kinase inhibitors, can also target the VEGF receptor, but once again trials have generally involved heavily pretreated patients with advanced disease, perhaps masking the true benefit of these novel drugs. Studies continue to elucidate whether angiogenic markers in tumors may be of prognostic and predictive value in the future.

Just as the expression of certain receptors can guide our treatments, so too can their absence. A number of different pathological subclasses of breast cancer have been identified based on gene-expression profiles. Of particular interest are triple-negative breast cancers - such as breast cancers that do not express ER, PgR or 
HER2. This subtype comprises approximately $15-20 \%$ of breast cancers, and the majority of triple-negative cancers have basal-like expression profiles, expressing cytokeratins that are normally found in the cell layer lying closest to the epithelial basement membrane [16]. Similar markers are also expressed in BRCA1associated cancers. Tissue microarray studies have shown a high rate of EGFR overexpression in these triple-negative cancers [17], and c-kit expression is also higher. Interestingly, these tumors are sensitive to chemotherapy, and the pCR rate may be higher in these cancers than some of the other subtypes. However, they have a high risk of relapse in contrast to other cases with pCRs. Evidence is now emerging that these cancers may be more sensitive to DNA-damaging agents, such as platinum-based regimens, as these tumors have deficient DNArepair mechanisms. As EGFR is also present in approximately $60 \%$ of these tumors, combining platinum-based treatment with EGFR inhibitors, such as gefitinib, could be an attractive therapeutic option.

In the future, gene-expression profiling may aid our decision-making when trying to identify particular patients who will most benefit from treatment. However, 'prognosis profiling' carries with it its own limitations. Oncotype $\mathrm{Dx}^{\mathrm{TM}}$ (Genomic Health, Inc., CA, USA), for example, is an assay that includes 16 tumor-related genes and five reference genes that generate a quantitative 'risk of recurrence' score [18]. Care must be taken when using these tools to help us decide about treatment, as the beneficial data they provide over and above ER, PgR and HER2 may be limited. Many of these assays are based on molecular profiling of a specific group of patients - what may be predictive in premenopausal, ER and lymph-node-negative patients may not apply in a post-menopausal, ER-positive patient. Although useful, there is currently insufficient data and evidence to base our clinical decisions purely on these assays.

Alongside the well established predictive markers such as ER, PgR and HER2, we still require better tools to correctly identify patients that will truly benefit from chemotherapy. Despite recent advances, we are still unable to predict the patients that may respond to treatment, particularly to some of the novel drugs such as EGFR inhibitors and antiangiogenics. Further biomarkers are needed if we are to truly realize the potential for improving our prognostic and predictive tools in the treatment of breast cancer. As we now know that receptors expressed by a tumor may change, we are currently studying those on the surface of circulating tumor cells, obtained in a single blood test, in order to guide our therapeutic strategies [19]. This creates the possibility of treating a patient according to their expression of receptors at that time.

\footnotetext{
Financial \& competing interests disclosure

The authors have no relevant affiliations or financial involvement with any organization or entity with a financial interest in or financial conflict with the subject matter or materials discussed in the manuscript. This includes employment, consultancies, honoraria, stock ownership or options, expert testimony, grants or patents received or pending, or royalties.

No writing assistance was utilized in the production of this manuscript.
}

\section{Bibliography}

1. Ravdin PM, Green S, Dorr TM et al.: Prognostic significance of progesterone receptor levels in estrogen receptor-positive patients with metastatic breast cancer treated with tamoxifen: results of a prospective Southwest Oncology Group study. J. Clin. Oncol. 10(8), 284-1291 (1992).

2. Burcombe RJ, Makris A, Richman PI et al.: Evaluation of ER, PgR, HER-2 and Ki-67 as predictors of response to neoadjuvant anthracycline chemotherapy for operable breast cancer. Br. J. Cancer 92(1), 47-155 (2004).

3. Tewari M, Krishnamurthy A, Shukla HS: Predictive markers of response to neoadjuvant chemotherapy in breast cancer. Surg. Oncol. 17(4), 301-311 (2008).
4. Rosenberg CL: Polysomy 17 and HER-2 amplification: true, true, and unrelated. J. Clin. Oncol. 26(30), 4856-4858 (2008).

5. Pritchard KI, Messersmith H, Elavathil L, Trudeau M, O’Malley F, Dhesy-Thind B: HER-2 and topoisomerase II as predictors of response to chemotherapy. J. Clin. Oncol. 26(5), 736-744 (2008).

6. Hayes DF, Thor AD, Dressler LG et al.: HER 2 and response to paclitaxel in node-positive breast cancer. N. Engl. J. Med. 357(15), 1496-1506 (2007).

7. Ellis MJ, Coop A, Singh B et al.: Letrozole is more effective neoadjuvant endocrine therapy than tamoxifen for ErbB-1- and/or ErbB-2positive, estrogen receptor-positive primary breast cancer: evidence from a Phase III randomized trial. J. Clin. Oncol. 19(18), 3808-3816 (2001).
8. Smith IE, Dowsett M, Ebbs SR et al.: Neoadjuvant treatment of postmenopausal breast cancer with anastrozole, tamoxifen, or both in combination: the Immediate Preoperative Anastrozole, Tamoxifen, or Combined With Tamoxifen (IMPACT) multicenter double-blind randomized trial. J. Clin. Oncol. 23(22), 5108-5116 (2005).

9. Prat A, Baselga J: The role of hormonal therapy in the management of hormonal-receptor-positive breast cancer with co-expression of HER2. Nat. Clin. Pract. Oncol. 5(9), 531-542 (2008).

10. Campos SM: Anti-epidermal growth factor receptor strategies for advanced breast cancer. Cancer Invest. 26(8), 757-768 (2008). 
11. Lynch TJ, Bell DW, Sordella R et al:: Activating mutations in the epidermal growth factor receptor underlying responsiveness of non-small-cell lung cancer to gefitinib. N. Engl. J. Med. 350 (21), 2129-2139 (2004).

12. Geyer CE, Forster J, Lindquist D et al.: Lapatinib plus capecitabine for HER2positive advanced breast cancer. $N$. Engl. J. Med. 355(26), 2733-2743 (2006).

13. Foekens JA, Peters HA, Grebenchtchikov N et al.: High tumor levels of vascular endothelial growth factor predict poor response to systemic therapy in advanced breast cancer. Cancer Res. 61(14), 5407-5414 (2001).

14. Miller K: E2100: a Phase III trial of paclitaxel versus paclitaxel/bevacizumab for metastatic breast cancer. Clin. Breast Cancer 3(6), 421-422 (2003).
15. Schneider BP, Sledge GW: Drug insight: VEGF as a therapeutic target for breast cancer. Nat. Clin. Pract. Oncol. 4(3), 181-189 (2007).

16. Irvin WJ Jr, Carey LA: What is triplenegative breast cancer? Eur. J. Cancer 44(18), 2799-2805 (2008).

17. Colleoni M, Viale G, Zahrieh D et al.: Expression of ER, PgR, HER1, HER2, and response: a study of preoperative chemotherapy. Ann. Oncol. 19(3), 465-472 (2008).

18. Marchionni L, Wilson RF, Wolff AC et al: Systematic review: gene expression profiling assays in early-stage breast cancer. Ann. Intern. Med. 148(5), 358-369 (2008).

19. Payne RE, Yagüe E, Slade MJ et al.: Measurements of EGFR expression on circulating tumor cells are reproducible over time in metastatic breast cancer patients. Pharmacogenomics 10(1), 51-57 (2009).

\section{Affiliations}

- Ursula B McGovern

Imperial College Healthcare NHS Trust, Charing Cross Hospital, Department of Medical Oncology, 1st Floor, East Wing, Fulham Palace Road, London, UK Tel.: +44 2087468295 Fax: +442088461433 u.mcgovern@imperial.ac.uk Justin Stebbing Imperial College Healthcare NHS Trust, Charing Cross Hospital, Department of Medical Oncology, 1st Floor, East Wing, Fulham Palace Road, London, UK justinstebbing@gmail.com 